\title{
Development of electrical powerless system for blood separation and coagulation time measurement
}

\author{
Jia-Cheng Lin', Thierry Burnouf ${ }^{2,3}$, Yu-Wen Wu ${ }^{2}$, Yu-Jui Fan ${ }^{1,3}$ \\ ${ }^{1}$ School of Biomedical Engineering, College of Biomedical Engineering, Taipei Medical University, Taipei, Taiwan; ${ }^{2}$ Graduate Institute of Biomedical \\ Materials and Tissue Engineering, College of Biomedical Engineering, Taipei Medical University, Taipei, Taiwan; ${ }^{3}$ International PhD Program in \\ Biomedical Engineering, College of Biomedical Engineering, Taipei Medical University, Taipei, Taiwan \\ Contributions: (I) Conception and design: JC Lin, YJ Fan; (II) Administrative support: YJ Fan; (III) Provision of study materials or patients: YJ Fan, \\ T Burnouf, YW Wu; (IV) Collection and assembly of data: JC Lin, YJ Fan; (V) Data analysis and interpretation: JC Lin, YJ Fan; (VI) Manuscript \\ writing: All authors; (VII) Final approval of manuscript: All authors. \\ Correspondence to: Yu-Jui Fan. School of Biomedical Engineering, College of Biomedical Engineering, Taipei Medical University, No. 250, Wu-Hsing \\ Street, Taipei 11031, Taiwan. Email: Ray.yj.fan@tmu.edu.tw.
}

Background: In recent years, developing low-cost point-of-care diagnostics for the areas where the spread of disease is fast, and where resources are limited, is becoming a vital topic of research. In this study, a blood separation and the determination of blood condition without using power was studied. A powerless centrifuge using finger spinner for Red blood cell separation and a self-powered plasma coagulation time measuring device have been developed. The finger spinner was designed and made by $3 \mathrm{D}$ printer. After cells are separated, the plasma will be obtained. A self-powered micro-channel device which is able to generate electrical signals by using triboelectric phenomenon was developed. When fluid flows through a solid surface, potential difference between the liquid and the solid surface is generated by friction. The device can be used to determine the coagulation time of the plasma. When the coagulation happens, the induction potential will be decreased. Combined the finger spinner centrifuge and a self-powered micro-channel, a blood condition detecting system without power can be obtained.

Methods: This study used 3D computer aided design (CAD) to design the finger spinner, and used 3D printer to make a four-arm finger spinner centrifuge to achieve blood separation without electricity. Then we used the triboelectric effect between solid and liquid, and combined with the design of the micro-channel and the gold electrode to form a system that can detect self-powered electrical signal when plasma flow in microfluidic channel. First, the sample was loaded in a syringe, and a stable flow rate is supplied through a syringe pump into the device. When the fluid flow in the micro-channel flow, because of the triboelectric effect, the charge is generated and accumulated at a thin dielectric layer covering on the electrode and channel wall. The voltage difference between electrode and the fluid was measured. In this study, we use different kinds of fluid to test, such as deionized water, glycerin with different concentrations, plasma, and plasma mixed thrombin. In the end, we collect different electrical signals by changing different viscosity to summarize the utility of the measurement wafer.

Results: The results show that the four-arm finger spinner centrifuge has a better effect to centrifuge than the dual-arm finger spinner centrifuge. Through the result of glycerin with different concentrations and plasma mixed thrombin, the increase in viscosity and plasma coagulation leads the decrease of the voltage difference.

Conclusions: In this study, the increase of the moment of inertia by weight design was investigated to improve the centrifuge effect for blood separation. For triboelectric effect at liquid-solid interface, the increase of the viscosity resulted in decrease of voltage difference. When plasma start to coagulate, the viscosity of plasma will decrease, and the voltage difference decrease. 
Keywords: Self-power; blood separation; plasma; blood coagulation time

Received: 15 March 2019; Accepted: 23 April 2020; Published: 08 June 2020.

doi: 10.21037/ht-19-11

View this article at: http://dx.doi.org/10.21037/ht-19-11

\section{Introduction}

To determine blood physiological status of hospitalized patients, rapid and efficient centrifugation of blood and measurement of coagulation time is vital. Unexpected and unconstrained coagulation of blood may cause blood clots during surgery, leading to serious medical complications such as thromboembolism and arteriosclerosis (1). Conversely, if the patient is given anticoagulants too early, serious problems such as massive bleeding may occur after surgery (2). Therefore, it is highly relevant to be able to determine the blood coagulation potential during the operation. However, the current way to measure the coagulation time is through blood sample analysis within cuvette (1) or using optical instruments to measure the signal of the scattered light output, and obtain the coagulation time by the maximum difference obtained (3). Although these methods may be effective, they have drawbacks which need to be overcome. Those drawbacks include the requirement to have typically large equipment that consumes a lot of energy.

Traditional mechanics can use hand-powered paper centrifuge (4), but is not very stable. Therefore, we used the concept of moment of inertia to make a finger spinner centrifuge to achieve the effect of blood separation without power.

Recent new inventions make it easy to carry and use in homecare. It measures the rate of the change, or the value, of capacitance or impedance between two electrodes situated on either sides of the microchannel (5). But this invention only observes impedance, it is not comprehensive for the factors to measure coagulation time. Thus, we combined the ideal of triboelectric effect, using the voltage difference signal generated by the friction between the solid and the fluid (6), to discuss the factors affecting the blood coagulation time, and to attempt to modify the surface of the microchannel (7) to enhance the effect and get larger signal.

The experimental process is showed in Figure 1. The developed finger spinner was used to separate the blood components, and the plasma was loaded into the sensing device by syringe for testing the coagulation time. A stable flow rate is supplied through a syringe pump into the microfluidic device. The induction electrical voltage generated by the triboelectric effect occurs when the fluid flows through the electrode, and the voltage difference between the fluid and the electrode was measured by using a voltmeter.

\section{Methods}

\section{Fabrication of finger spinner and blood separation}

We used a $3 \mathrm{D}$ computer aided design (CAD) to design our finger spinner. In order to allow the centrifuge tube to be embedded in the finger spinner, grooves for setting the centrifuge tubes on the rotating arms were designed (Figure 2). Figure $2 A$ is our first design, which is a dual-arm finger spinner.

A four aim finger spinner, two for setting capillaries and two for counter weight by embedding bearing. By increasing the weight, the moment of inertia can be increased and the problem of the weight can be solved. Therefore, a fourarm finger spinner centrifuge was designed (Figure 2B). We designed two holes in the additional rotating arms to place the ball bearings to adjust the weight.

Then we used 3D printer to fabricate the finger spinner, as shown in Figure $2 C$ and $D$. After polishing the surface, the ball bearing was embedded in the middle of the finger spinner. In addition, ball bearings were embedded in the rotating arms of the four-arm finger spinner to adjust the weight, as shown in Figure $3 A$ and $B$.

Then we used four-arm finger spinner centrifuge to separate the blood. First, through the capillary principle, the blood is collected by capillary tube. Then we could use vaseline to close both ends of the opening site and put it into our finger spinner centrifuge. Finally, we could apply an external force to start the finger spinner centrifuge to separate the blood.

\section{Self-powered coagulation time measurement device}

A self-powered micro-channel device for measuring blood coagulation time was developed. The triboelectric effect between solid and liquid was used. The microchannel 


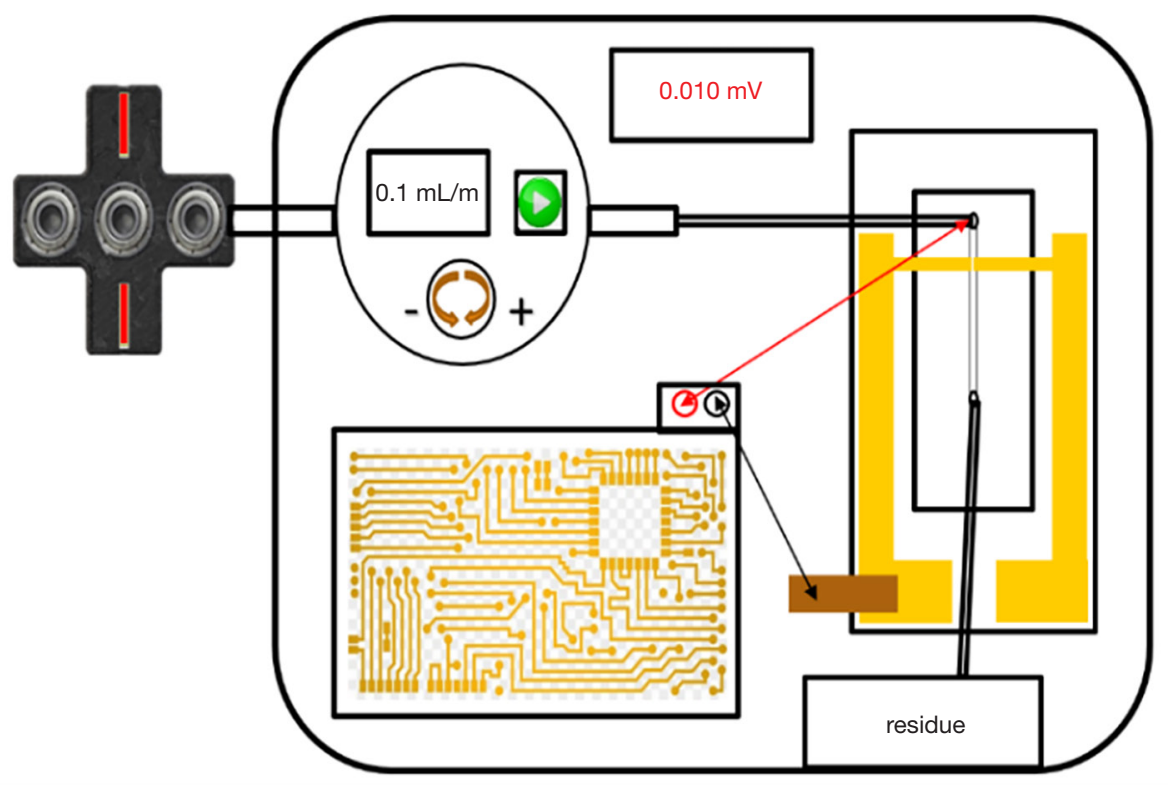

Figure 1 The device schematic. It conclude (I) centrifuge of finger spinner to separate the blood to get plasma, (II) vacuum pump to get a stable flow, (III) microchannel wafer, and (IV) circuit board to read the signal.
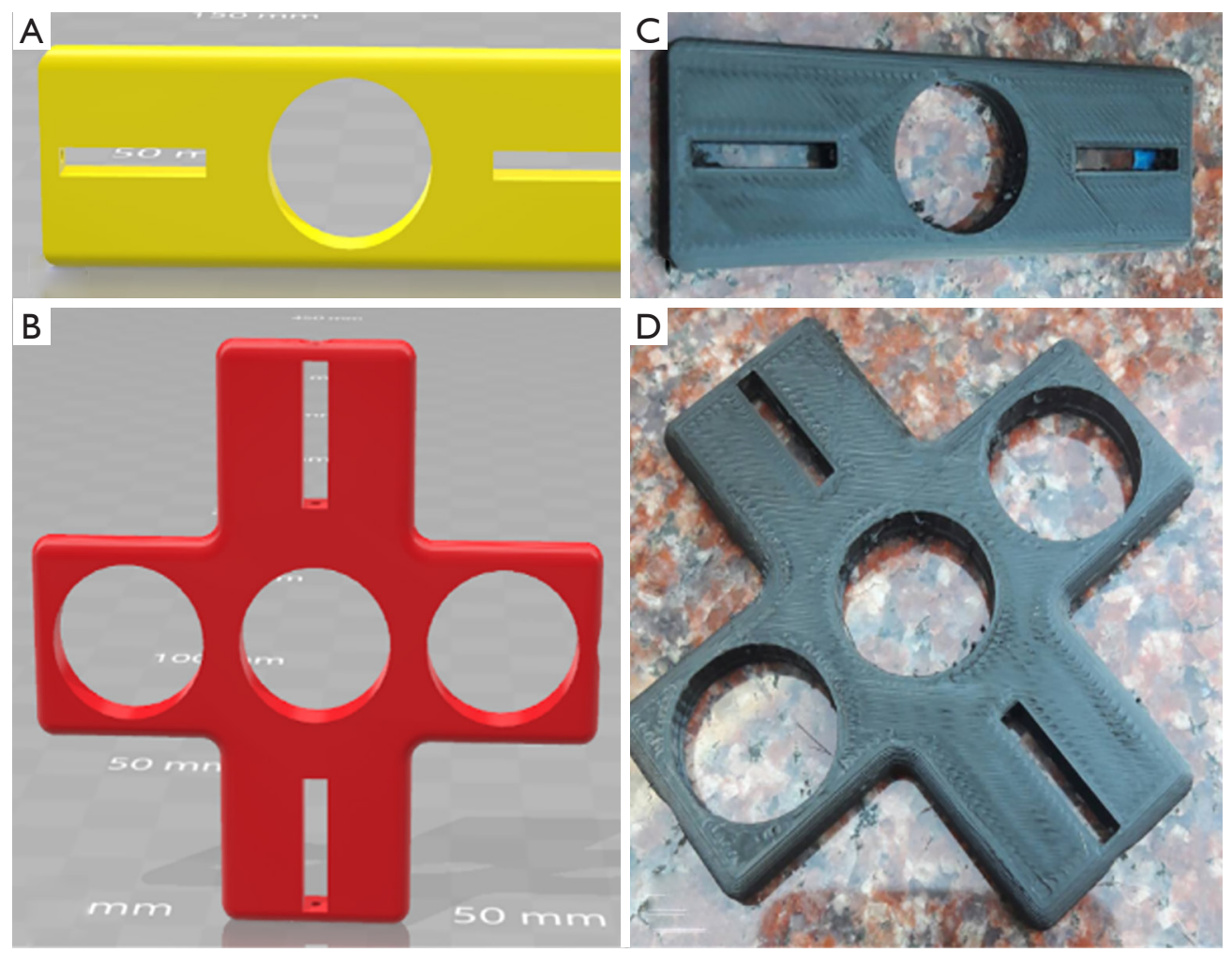

Figure $23 \mathrm{D}$ computer aided design (CAD) for $(\mathrm{A})$ dual-arm and (B) four-arm finger spinner. 3D printing product for (C) dual-arm and (D) four-arm finger spinner. 

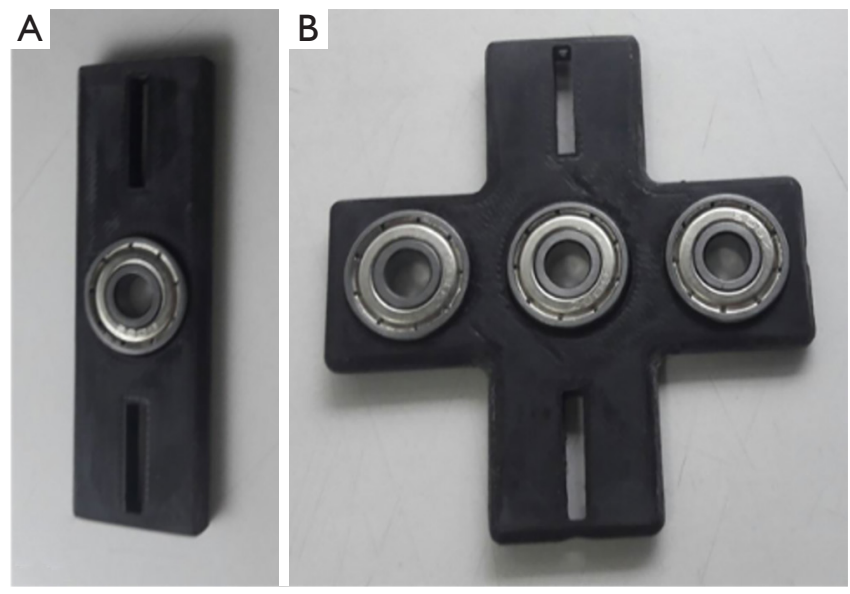

Figure 3 Development of finger spinner. (A) Dual-arm finger spinner and (B) four-arm finger spinner designed and fabricated by $3 \mathrm{D}$ computer aided design $(\mathrm{CAD})$ and $3 \mathrm{D}$ printer.

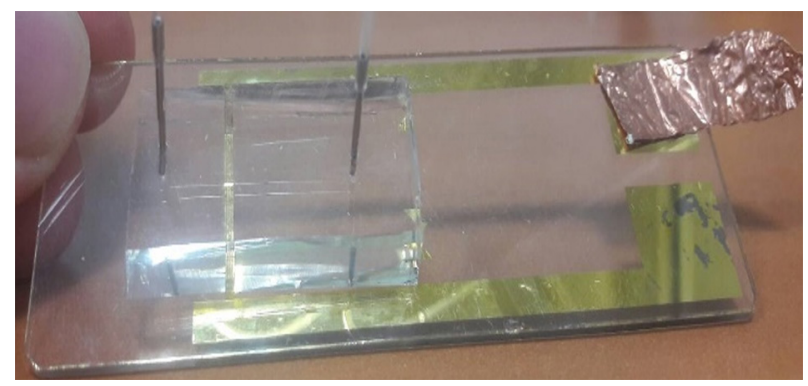

Figure 4 Self-powered coagulation time measurement device includes microfluidic channel with gold electrode covering with Cytop.

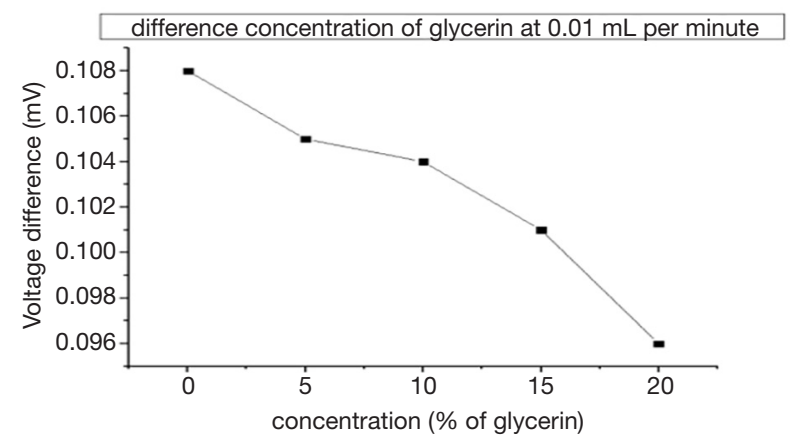

Figure 5 Concentrations of glycerin versus voltage difference.

and gold electrode to form a system was designed. In the microfluidic channel, the Cytop was coated in the channel wall as a dielectric layer (Figure 4).
The sample was injected into microfluidic device with stable flow-rate. When the fluid flew through the electrode covered with Cytop, the charges were generated and accumulated at the dielectric layer. By measuring the potential difference between electrode and fluid, the properties of fluids could be determined.

In this study, we used different kinds of fluid to test, such as deionized water, glycerin with different concentrations, plasma, and plasma mixed thrombin. In the end, we collected different electrical signals by changing different viscosity to summarize the utility of the measurement wafer.

\section{Results}

The four-arm finger spinner had two additional rotating arms embedded ball bearings. It can solve the problem of enough weight, and it still can reach 1,000 rpm. After experiment test, blood could be separated in 10 minutes by the four-arm finger spinner centrifuge.

For measuring the blood coagulation time, the viscosity of fluids versus potential difference was carried out by using different volume ratio of glycerol and deionized water (Figure 5). The real-time potential difference of the human plasma mixing with thrombin in the device was achieved (Figure 6).

The results showed that when the flow-rate of $0.01 \mathrm{~mL} / \mathrm{min}$ was applied, and the glycerol concentration was given from $0 \%$ to $20 \%$, the potential differences decreased from 0.108 to $0.096 \mathrm{mVDC}$ (Figure 5). When the viscosity was raised by using a higher concentration of glycerin, the voltage difference decreased. In addition, in the plasma test, we found that when we mixed plasma at $\mathrm{pH} 7$ with thrombin at $0.03 \mathrm{U} / \mathrm{mL}$, the plasma began to coagulate at 3 minutes and was fully clogged in 7 minutes (Figure 6). Comparing the plasma without thrombin, the potential difference decreased dramatically, and after 7 minutes, the potential difference was stable at a low value.

\section{Discussion}

Blood-related tests used to require substantial medical resources. From the sample acquisition to the analysis, instruments such as centrifuges and blood condition analyzers are needed. These instruments are not only huge and expensive, but they also consume lots of energy. For these reasons, their use in low-resources countries is often affordable, especially outside the capital city.

In comparison to conventional blood separation 


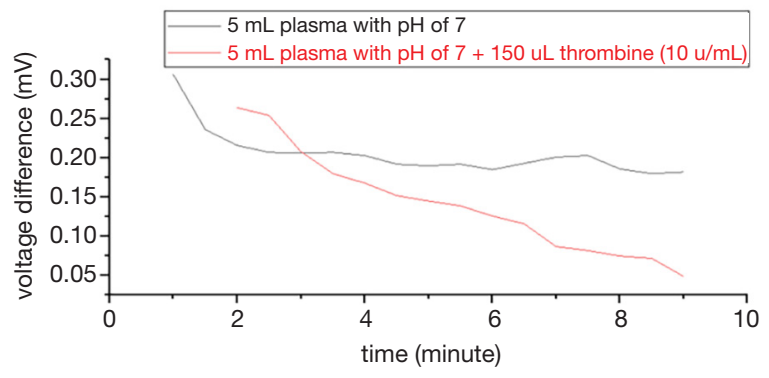

Figure 6 Comparison to mix plasma with $\mathrm{pH}$ of 7 and thrombin at $0.03 \mathrm{U} / \mathrm{mL}$ or not, the potential difference decreased dramatically at the test of the plasma w/o thrombin.

methods, our finger spinner features some advantages of being cheap, easy to carry, and not consuming electricity. Although the centrifugal efficiency is not necessarily better, but it still can separate blood, providing a better choice in areas lacking medical resources.

In the measurement of the blood coagulation time, our device works by measuring the voltage difference. In terms of accuracy, the optical detection used by the blood condition analyzer may be better, but our device can reduce the blood consumption and it is faster and easy to test. Therefore, it can achieve more effective rapid detection that is crucial for the safety of surgical procedures.

Through the experiment, we demonstrate the effects on electrical signal of some coagulation-related fluid properties, so we may be able to apply the technology to other clinical test, such as PT and APTT test in the future.

\section{Conclusions}

In this study, a finger spinner centrifuge was designed to separate the blood by moment of inertia without power. In addition, a self-powered plasma coagulation time measurement device was developed by using triboelectric effect through the solid and the fluid friction. We found that the lower viscosity resulted in larger potential difference. Therefore, the coagulation time can be determined by comparing the potential difference of the plasma without the thrombin in the same flow-rate.

\section{Acknowledgments}

We would also like to thank the NEMS Research Center, National Taiwan University for facility support.

Funding: This work was supported by the Ministry of
Science and Technology of Taiwan under grant numbers MOST 108-2911-I-038-504, and 107-2221-E-038-018.

\section{Footnote}

Data Sharing Statement: Available at available at http:// dx.doi.org/10.21037/ht-19-11

Conflicts of Interest: All authors have completed the ICMJE uniform disclosure form (available at http://dx.doi. org/10.21037/ht-19-11). The authors have no conflicts of interest to declare.

Ethical Statement: The authors are accountable for all aspects of the work in ensuring that questions related to the accuracy or integrity of any part of the work are appropriately investigated and resolved. The study was conducted in accordance with the Declaration of Helsinki (as revised in 2013). This study was approved by the institutional review board of the study hospital, and all volunteers provided informed consent before being allowed to participate (No. 20180620, 2018/06/20, TMU-Joint Institutional Review Board).

Open Access Statement: This is an Open Access article distributed in accordance with the Creative Commons Attribution-NonCommercial-NoDerivs 4.0 International License (CC BY-NC-ND 4.0), which permits the noncommercial replication and distribution of the article with the strict proviso that no changes or edits are made and the original work is properly cited (including links to both the formal publication through the relevant DOI and the license). See: https://creativecommons.org/licenses/by-nc-nd/4.0/.

\section{References}

1. Cusak R, Laduca FM, Samo RJ. Blood Coagulation Time Test Apparatus And Method. U.S. Patent No. 5372946.

2. van der Meer FJ, Rosendaal FR, Vandenbroucke JP, et al. Bleeding complications in oral anticoagulant therapy. An analysis of risk factors. Arch Intern Med 1993;153:1557-62.

3. Uji SK, Kyoto MY. Method And System For Measuring Blood Coagulation Time. U.S. Patent No. 4252536.

4. Bhamla MS, Benson B, Chai C, et al. Hand-powered ultralow-cost paper centrifuge. Nature Biomedical Engineering 2017;1:0009.

5. Stiene M, Richter T, McAleer J, et al. Device for 
Measuring Blood Coagulation And Method Thereof. U.S. Patent No. 7005857 B2.

6. Li X, Yeh MH, Lin ZH, et al. Self-Powered Triboelectric Nanosensor for Microfluidics and Cavity-Confined

doi: $10.21037 /$ ht-19-11

Cite this article as: Lin JC, Burnouf T, Wu YW, Fan YJ. Development of electrical powerless system for blood separation and coagulation time measurement. Health Technol 2020;4:2.
Solution Chemistry. ACS Nano 2015;9:11056-63.

7. Fan B, Bhattacharya A, Bandaru PR. Enhanced voltage generation through electrolyte flow on liquid-filled surfaces. Nat Commun 2018;9:4050. 